\title{
A Simple Chaotic Wien Bridge Oscillator with a Fractional-Order Memristor and Its Combination Synchronization for Efficient Antiattack Capability
}

\author{
Anitha Karthikeyan, ${ }^{1}$ Karthikeyan Rajagopal $\left(D,{ }^{2}\right.$ Victor Kamdoum Tamba, ${ }^{3}$ Girma Adam, ${ }^{4}$ \\ and Ashokkumar Srinivasan ${ }^{2}$ \\ ${ }^{1}$ Ton Duc Thang University, Ho Chi Minh City, Vietnam \\ ${ }^{2}$ Center for Nonlinear Systems, Chennai Institute of Technology, Malayambakkam, India \\ ${ }^{3}$ Department of Telecommunication and Network Engineering, IUT-Fotso Victor of Bandjoun, University of Dschang, \\ P. O. Box: 134, Bandjoun, Cameroon \\ ${ }^{4}$ Center for Nonlinear Dynamics, Defence University, Bishoftu, Ethiopia
}

Correspondence should be addressed to Karthikeyan Rajagopal; rkarthiekeyan@gmail.com

Received 1 September 2020; Revised 7 October 2020; Accepted 12 February 2021; Published 1 March 2021

Academic Editor: Chun-Biao Li

Copyright (C 2021 Anitha Karthikeyan et al. This is an open access article distributed under the Creative Commons Attribution License, which permits unrestricted use, distribution, and reproduction in any medium, provided the original work is properly cited.

\begin{abstract}
Memristor-based oscillators are of recent interest, and hence, in this paper, we introduce a new Wien bridge oscillator with a fractional-order memristor. The novelty of the proposed oscillator is the multistability feature and the wide range of fractional orders for which the system shows chaos. We have investigated the various dynamical properties of the proposed oscillator and have presented them in detail. The oscillator is then realized using off-the-shelf components, and the results are compared with that of the numerical results. A combination synchronization scheme is proposed which uses more than one driver systems to synchronize with one response system. Indeed, we use two different techniques where the first one consists of splitting the transmitted signals into two parts where each part is loaded in different drive systems, while the second one consists of dividing time into different intervals and loading the signals in different drive systems. Such techniques improve the antiattack capability of the systems when used for secure communication.
\end{abstract}

\section{Introduction}

Versatile dynamical behaviors observed from memristorbased chaotic circuits obviously challenge the scientific community. After the physical realization of the memristor by HP Labs, many real-time circuits were designed for different kinds of applications. On the contrary, complex systems which hold intricate properties, much sensitive to initial conditions and influenced by history of variable states, demand memristor-based mimic models. Sufficient literature studies were identified for extracting and investigating special properties such as coexistence of multiple attractors $[1-5]$, antimonotonicity $[6,7]$, hidden attractors $[3,8-11]$, and time delays $[12,13]$. The challenges are handled effectively, while implementing those models in real-time circuits $[1,3,8,10,11,14]$.

A horde of physical phenomena holds fractional-order description; therefore, the differential equations formulated to analyze its dynamical nature need to be treated with the fractional-order form. Noninteger order formulation provides a more accurate model of physical systems than integer calculus do. A variety of applications [15-21], both in one dimension and in multidimensional space, especially nonlinear behaviors are modelled with fractional-order differential equations in order to reveal the characteristics in the complex scenario. In [22], fractional time derivatives-based oscillators are formulated using the Riemann-Liouville method. A comparative study was carried out on linearly damped 
oscillator equations, and the fractional-order treatment showed interesting phenomena over integer-order treatment [23].

The characteristics of nonlinearity present in the memristor element leads the memristor-based circuits to generate a chaotic signal easily. The presence of a "pinched hysteresis loop" in the current-voltage characteristics of memristive system shows its nonlinear behavior. The principle of fractional calculus is based on the memory property of the fractional-order integral or derivative. Hence, the relation between fractional calculus and memristor is clear enough to understand, and the memristor can be extended to the fractional order as well. In [24], a fractional-order memristor is modelled for Chua's oscillator, and it successfully showed the chaotic behaviors. In [25], various fractional-order circuit elements were modelled and analyzed. Fractional-order generalized memristor-based chaotic systems are modelled and analyzed with circuit implementation [26]. Detailed analysis on fractional memristors and their intricate behaviors and influence on circuits are analyzed, and simple fractional-order circuits are also discussed in $[27,28]$. In the last decade, many chaotic oscillators were formulated using memelements especially the memristor [29-32].

In a general Wien bridge oscillator, an operational amplifier is connected parallel to RC and series RC networks. An attempt is made to replace the resistor in parallel configuration with a memristor in [33-35] and come up with a strange nonchaotic attractor. A fractional-order memristorbased hyperchaotic Wien bridge oscillator circuit is modelled using the Adomian Decomposition method, and analysis found that compared with the integer order form, the fractional-order form revealed many intricate properties like coexisting of multiple attractors [36]. In [37], analysis of dynamical behaviors and realization of a fractional-order memristor-based chaotic circuit is carried out.

The memristor is believed to be the essential for mimicking the neuron network of the artificial brain [38], and the configuration of the nervous system is physically closer to the fractal dimensions $[39,40]$. Henceforth, the research studies on the fractional-order memristive systems have great impact for the realization of the artificial intelligence. It is also necessary to do a detailed dynamical analysis for the memristive chaotic circuit system. There was recently a memristor-based chaotic oscillator which comes from the classical Chua circuit and is devoted to weak signal detection application discussed in the literature [41], and a van der Pol-Duffing oscillator was proposed and analyzed its applications in weak signal detection [42]. It is to be noted that all these literatures are integer order models but a fractionalorder memristor can perfectly model the hysteresis and memory effects of a memristor compared to their integer order counterparts. Hence, in this paper, we propose a new fractional-order memristor-based Wien bridge oscillator.

\section{Absolute Memfractor Wien Bridge Oscillator (AMWO)}

The word memfractor denotes the fractional-order memristor and was proposed in [43]. By the definition of memristor, the $n^{\text {th }}$ order voltage-controlled fractional-order memristor (memfractor) can be described as

$$
\begin{gathered}
i=M_{f} V, \\
D^{q} \phi=f(\phi, V),
\end{gathered}
$$

where $i$ is the current flowing through the memristor, $V$ is the voltage across the memristor, and $\phi$ is the state variable. The new voltage-controlled fractional-order absolute memristor can be modelled as

$$
\begin{gathered}
i=M_{f} V, \\
D^{q} \phi=-C \phi-d V^{2},
\end{gathered}
$$

where $M_{f}=\alpha_{f}-\beta_{f}|\phi|$ is the absolute memristor. The equivalent circuit for the fractional-order absolute memristor is shown in Figure 1. We have used the fractionalorder integrator proposed in $[44,45]$ to design the circuit. Figure 1(a) shows the fractional-order memristor emulator, and Figure 1(b) shows the fractional-order integrator used in Figure 1(a).

Using the memfractor circuit, we can derive the following modified forms of (2) as follows:

$$
\begin{aligned}
i & =\left(\frac{1}{R_{j}}-\frac{R_{g}}{R_{j} R_{h}}|\phi|\right) V, \\
D^{q} \phi & =-\frac{1}{R_{i} C_{i}} \phi-\frac{1}{R C_{i}} V^{2} .
\end{aligned}
$$

We use memfractor model (3) to design a new absolute memfractor Wien bridge oscillator, as shown in Figure 2. In the circuit, we consider voltage across the capacitors $c_{a}$ and $c_{b}$ and the current through the memfractor $\left(M_{f}\right)$ as the three state variables. We have also shown the V-I characteristics of the memfractor using the simulation and experimental model in Figure 2 (lower).

Applying Kirchoff's law to the circuit shown in Figure 2, the mathematical model can be represented as

$$
\begin{aligned}
\dot{V}_{a} & =\frac{R_{b}}{C_{a} R_{a}}\left[\frac{V_{a}-V_{b}}{R_{c}}+M_{f} V_{a}\right], \\
\dot{V}_{b} & =\frac{V_{a}-V_{b}}{C_{b} R_{c}}, \\
D^{q} \phi & =-a_{3} \phi-a_{4} V_{a}^{2},
\end{aligned}
$$

where $M_{f}=\alpha_{f}-\beta_{f}|\phi|$. Let us define $x=V_{a}, y=V_{b}, z=\phi$, $R_{a}=R_{b}, a_{1}=R_{b} / C_{a} R_{a} R_{c}, a_{2}=R_{b} / C_{b} R_{a} R_{c}, \alpha=\alpha_{f} / C_{a}$, and $\beta=\beta_{f} / C_{a}$, and using these definitions in (4), the dimensionless model can be derived as

$$
\begin{aligned}
\dot{x} & =a_{1}(x-y)+M_{f} x, \\
\dot{y} & =a_{2}(x-y), \\
D^{q} z & =-a_{3} z-a_{4} x^{2} .
\end{aligned}
$$

We have used the predictor-corrector method $[46,47]$ to discretize the AMWO system (5), and the predict evaluate 


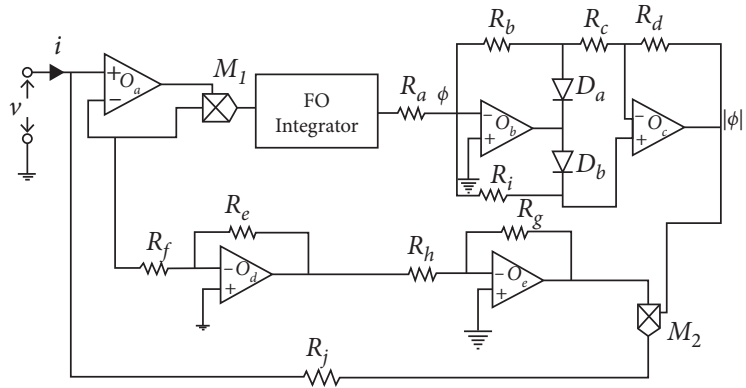

(a)

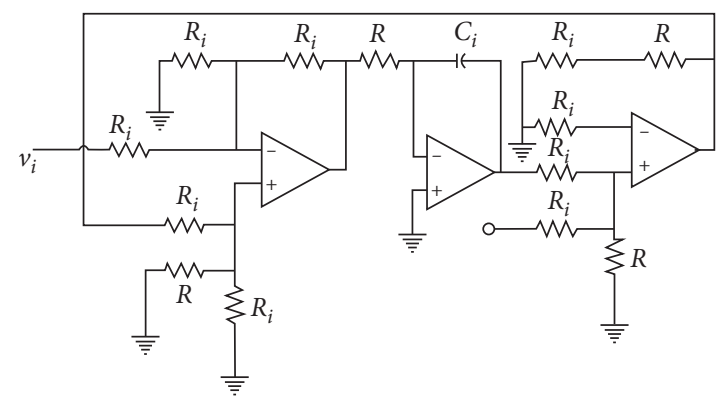

(b)

Figure 1: (a) Fractional-order absolute memristor emulator; (b) fractional-order integrator using first-order approximation.

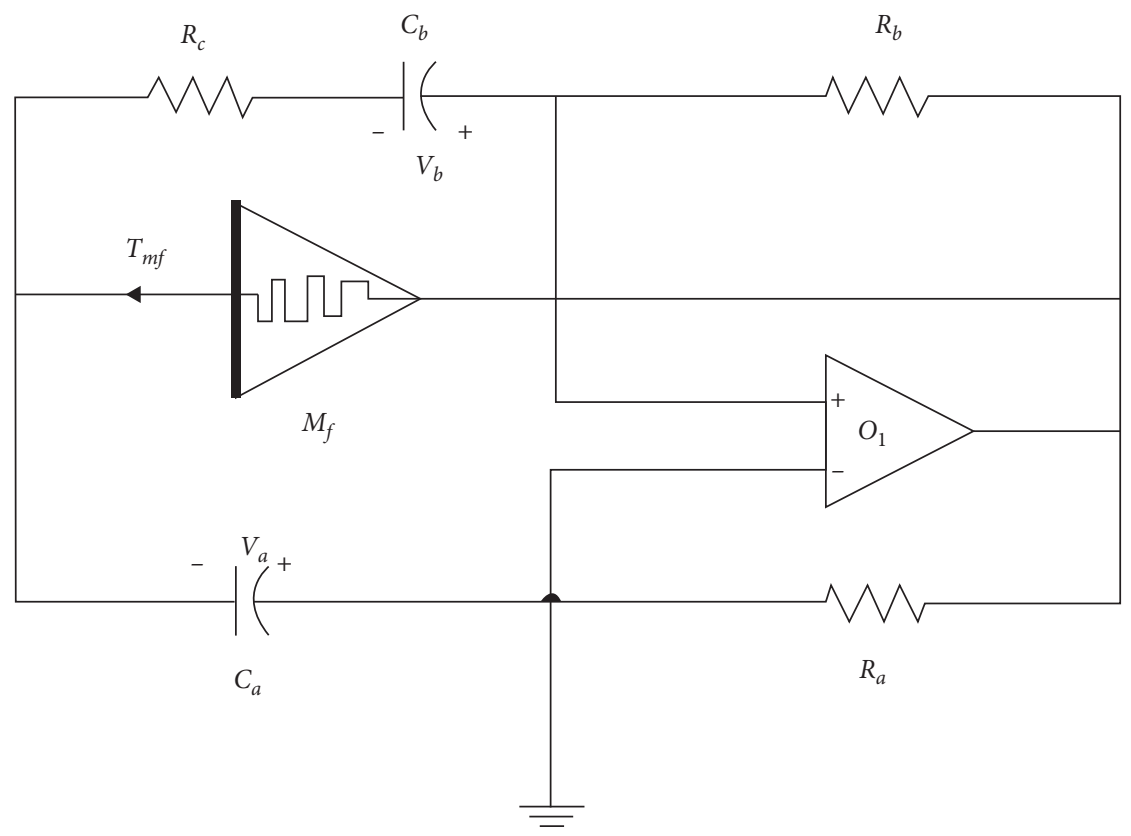

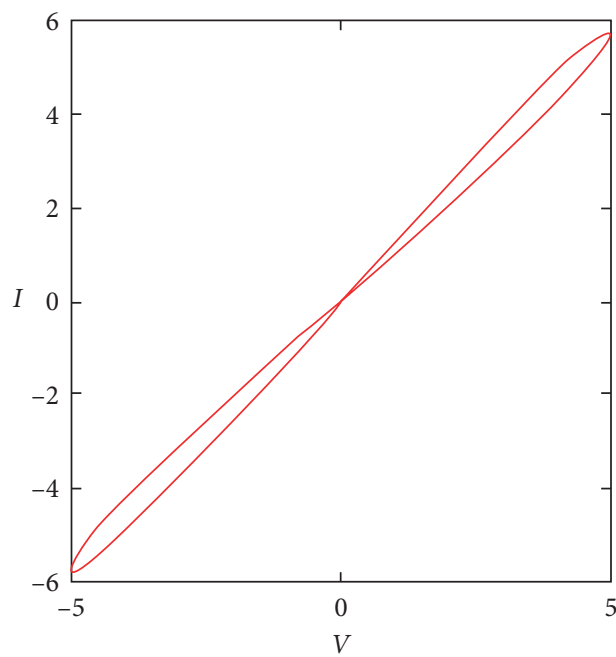

(a)

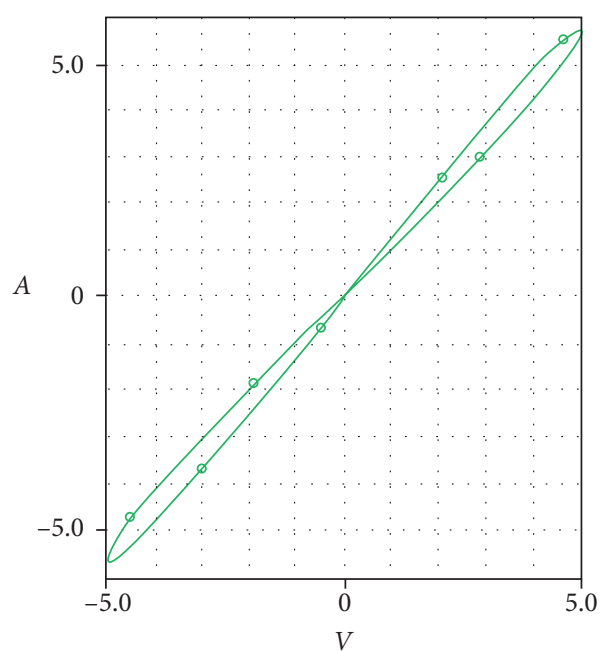

。 I

(b)

FiguRE 2: Upper: absolute memfractor Wien bridge oscillator; lower: the I-V characteristic of the memfractor for $q=0.95$ using (a) simulation and (b) Pspice circuit. 
correct evaluate (PECE) method of ABM studied in [48, 49] used. In order to derive the general model of the PECE $[46,47]$ method, the third-state variable of the AMWO with order $q$ is considered as

$$
D^{q} z=f(t, z), \quad 0 \leq t \leq T,
$$

where $z^{k}(0)=z_{0}^{k}$ for $k \in[0, n-1]$.

Equation (6) is similar to the Volterra integral equation $[23]$ as

$$
z(t)=\sum_{k=0}^{n-1} z_{0}^{k} \frac{t^{k}}{k !}+\frac{1}{\Gamma(q)} \int_{0}^{t} \frac{f(\tau, z)}{(t-\tau)^{1-q}} d \tau,
$$

which in equation (7), $h=T / N$, and $t_{n}=n h$ as $h \in[0, N]$. The discrete form of equation (8) can be defined as

$$
\begin{aligned}
z_{h}\left(t_{n+1}\right)= & \sum_{k=0}^{n-1} z_{0}^{(k)} \frac{t_{n}^{k+1}}{k !}+\frac{h^{q}}{\Gamma(q+2)} f\left(t_{n+1}, z_{h}^{p}\left(t_{n+1}\right)\right) \\
& +\frac{h^{q}}{\Gamma(q+2)} \sum a_{j, n+1} f\left(t_{j}, z_{h}\left(t_{j}\right)\right),
\end{aligned}
$$

where

$$
\begin{aligned}
a_{j, n+1} & =\left\{\begin{array}{c}
n^{q+1}-(n-q)(n+1)^{q+1}, \quad j=0, \\
-2(n-j+1)^{q+1}, \quad 1 \leq j \leq n, \\
1, \quad j=n+1,
\end{array}\right\} \\
z_{h}^{p}\left(t_{n+1}\right) & =\sum_{k=0}^{n-1} z_{0}^{(k)} \frac{t_{n}^{k+1}}{k !}+\frac{h^{q}}{\Gamma(2)} \sum_{j=0}^{n} b_{j, n+1} f\left(t_{j} z_{h}\left(t_{j}\right)\right), \\
b_{j, n+1} & =\frac{h^{q}}{q}\left((n-j+1)^{q}-(n-j)^{q}\right) .
\end{aligned}
$$

The error estimate is $e=\operatorname{Max}\left|z\left(t_{i}\right)-z_{h}\left(t_{i}\right)\right|=0\left(h^{p}\right)$ $(j=0$ to $N)$, where $p=\operatorname{Min}(2,1+q)$. For the numerical analysis of the AMWO system, we have used RK-4 to solve the first two state equations, while the third fractional-order state is solved using PECE derived in (8). System (5) shows the chaotic attractor for $a_{1}=4.5$, $a_{2}=5.5, a_{3}=0.4, a_{4}=1, \alpha=5, \beta=4$, and fractional order $q=0.98$. We have shown the $2 \mathrm{D}$ phase portraits, as shown in Figure 3, and the step size $h=0.01$. It is to be noted that most of the fractional-order literatures showed that having larger step size will require greater memory size. Hence, as said in literatures [21-24], the step size is taken as $h=0.01$.

\section{Dynamical Properties of the AMWO System}

In order to investigate the dynamical behavior of the AMWO system, we have used the tools like the equilibrium points and its stability, Lyapunov exponents, bifurcation plots, Lyapunov spectrum, Poincare map, basin of attractions, etc.

3.1. Stability of Equilibrium Points. The AMWO system has three equilibrium points as follows:

$$
\begin{aligned}
E_{1} & =[0,0,0], \\
E_{2,3} & =\left[ \pm \sqrt{\frac{\alpha a_{3}}{\beta a_{4}}}, \pm \sqrt{\frac{\alpha a_{3}}{\beta a_{4}}},-\frac{\alpha}{\beta}\right] .
\end{aligned}
$$

The generalized characteristic polynomial of the AMWO system is calculated using the relation $\operatorname{det}\left(\operatorname{diag}\left(\lambda, \lambda, \lambda^{0.9}\right)-\right.$ $\left.J_{E}\right)=0$ which is derived as

$$
\begin{aligned}
\lambda^{29} & +\lambda^{21}+(c+2) \lambda^{20}-b d \lambda^{19}+2 \lambda^{12}+(2 c-b d+1) \lambda^{11} \\
& -(b d+b c d) \lambda^{10}+a b \lambda^{9}+\lambda^{3}+(c-b d) \lambda^{2} \\
& +(a b-b c d) \lambda+a b c=0 .
\end{aligned}
$$

In parameter values $a_{1}=4.5, a_{2}=5.5, a_{3}=0.4, a_{4}=1$, $\alpha=5$, and $\beta=4$, the characteristic equation of the AMWO system for the equilibrium point $E_{1}$ is $\lambda^{29}+\lambda^{21}+2.4 \lambda^{20}+$ $4 \lambda^{19}+2 \lambda^{12}+2.2 \lambda^{11}-5.6 \lambda^{10}+27.5 \lambda^{9}$

$+\lambda^{3}-3.6 \lambda^{2}-29.1 \lambda-11=0$. The stability of the AMWO system can be defined by the following corollaries.

Corollary 1. The AMWO is asymptotically stable if

$$
q>\frac{2}{\pi} \arctan \left(\frac{|\operatorname{Im}(\lambda)|}{\operatorname{Re}(\lambda)}\right),
$$

for any $\lambda$ of the equilibrium points. In this case, the components of the state decay towards 0 like $t$.

The eigenvalues of the AMWO for the equilibrium points $E_{2,3}$ are plotted against the fractional order $q$, as shown in Figure 4, and it could be easily seen that, for $q>0.849$, the system is asymptotically stable.

Corollary 2. For the chaotic attractor to exist in the AMWO, the equilibrium points corresponding to the oscillations should exhibit instability. So, the necessary condition for the existence of the unstable equilibrium is

$$
\frac{\pi}{2 M}-\min _{i}\left\{\arg \left(\lambda_{i}\right)\right\} \geq 0
$$

where $\lambda_{i}$ are the roots of $\operatorname{det}\left(\operatorname{diag}\left(\lambda^{M q_{x}}, \lambda^{M q_{x}}, \lambda^{M q_{x}}\right)_{-} J_{E_{i}}\right)$ for each $E_{i}$.

Using Corollary 1 and Corollary 2, the AMWO has chaotic dynamics in $q>0.89$ for $E_{2,3}$.

3.2. Lyapunov Exponents. Lyapunov exponents (LEs) of the AMWO are derived using Wolf's algorithm [50] and the fractional-order predictor-corrector [49] solver fde12 [51] as the ode solvers [52]. The LEs for the AMWO system are calculated as $[0.475,0 .-2.26]$ for fractional order $q=0.98$. The Kaplan-Yorke dimension of the AMWO system is $D_{K Y}=2.21$.

3.3. Bifurcation. To investigate the parameter impact on the AMWO system, we derive the bifurcation plots. We considered two different bifurcation scenarios with the first one 


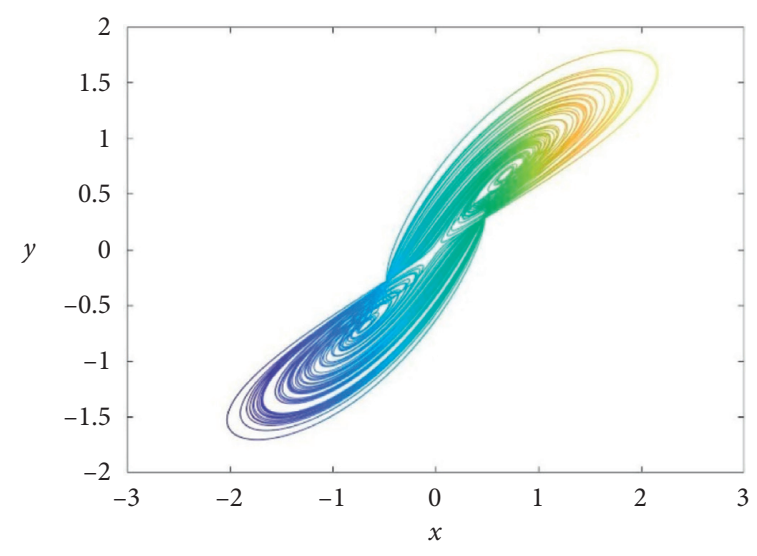

(a)

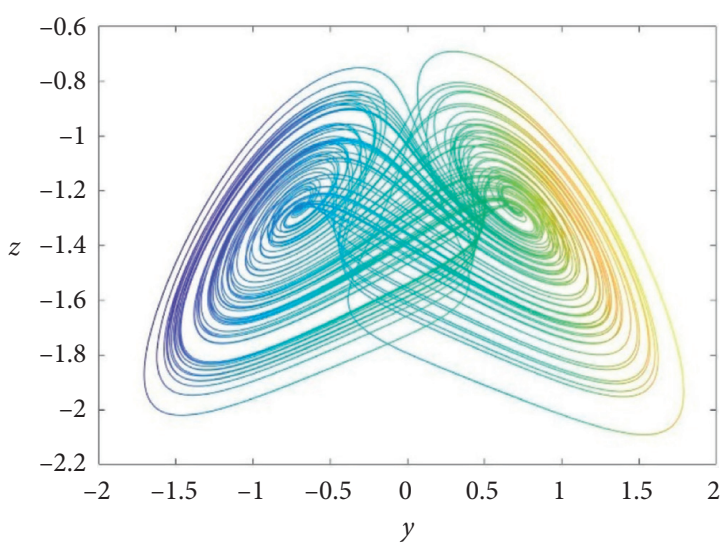

(b)

FIgURe 3: 2D phase portraits of AMWO system (5) for the initial conditions $[0.1 ; 0.1 ; 0.1]$.

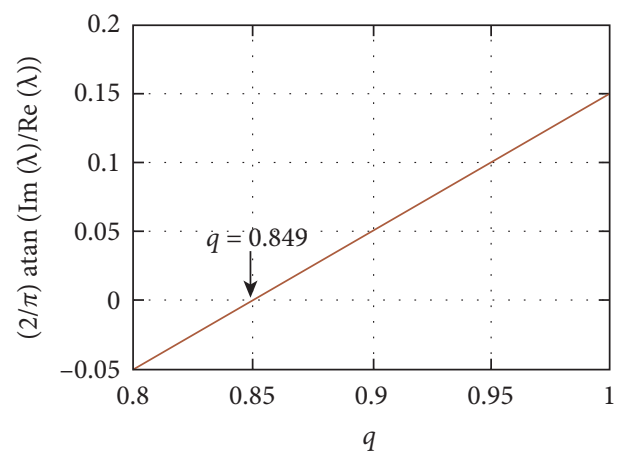

(a)

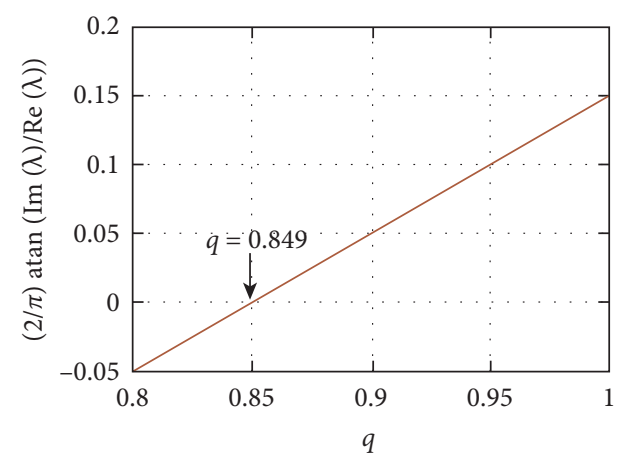

(b)

Figure 4: Condition for asymptotic stability of AMWO for the equilibriums $E_{2,3}$ ((a), (b)).

taking the parameter $\alpha$ as the control value and in the second one taking the fractional order as the control variable. We have used forward and backward continuation in which either the parameter is increased or decreased with reinitializing of the initial conditions in every iteration to the end values of state trajectories, as shown in Figure 5(a). We have also plotted the corresponding finite-time Lyapunov exponents (LEs) calculated using Wolf's algorithm [50] and the fractional-order predictor-corrector [49] solver fde12 [51] as the ode solvers [52], as shown in Figures 5(b) and 5(c). We could clearly see coexisting chaotic attractors for $57 \leq \alpha \leq 68$, coexisting period- 4 limit cycles for $68 \leq \alpha \leq 69$, coexisting period-2 limit cycles for $69 \leq \alpha \leq 78$, and coexisting period- 1 limit cycle for $78 \leq \alpha \leq 80$. The various coexisting attractors for different values of $\alpha$ at $q=0.98$ are shown in Figures 6(a)-6(c). The basin of attraction of the system in $x-z$ plane is shown in Figure 6(d).

In the second scenario, we have considered the fractional order of the AMWO system and have derived the bifurcation plot as seen in Figure 7(a). We could see a very narrow band of chaos for $0.845 \leq q \leq 0.847$ and a wide band of chaos for $0.85 \leq q \leq 1$. The LEs are computed and shown in Figure 7(a).

\section{Circuit Implementation}

In this section, we perform some Pspice-based circuit simulations in order to compare the results with those obtained numerically. The analogue circuit diagram modelling of the AMWO system (5) is provided in Figure 8. By using Kirchhoff's electric laws on the analogue circuit diagram of Figure 8, the corresponding circuit state equations can be expressed as

$$
\left\{\begin{array}{c}
C \frac{d V_{x}}{d t}=\frac{V_{x}}{R_{1}}-\frac{V_{y}}{R_{2}}+\frac{V_{x}}{R_{3}}-\frac{\left|V_{z}\right| V_{x}}{R_{4}}, \\
C \frac{d V_{y}}{d t}=\frac{V_{x}}{R_{5}}-\frac{V_{y}}{R_{6}}, \\
C_{0} \frac{d^{q} V_{z}}{d t^{q}}=-\frac{V_{x}^{2}}{R_{7}}-\frac{V_{z}}{R_{8}},
\end{array}\right\}
$$

where $V_{x}, V_{y}$, and $V_{z}$ are the output voltages of the operational amplifiers OP1, OP2, and OP3, respectively. The capacitor $C_{0}$ denotes the fractional-order unit circuit 

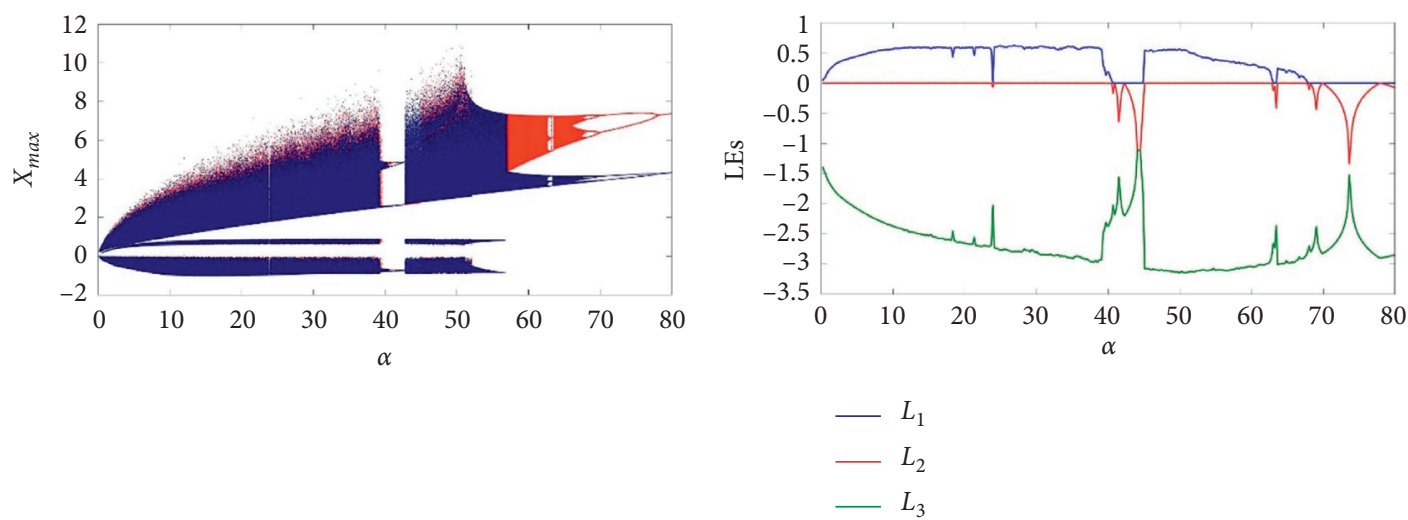

(a)

(b)

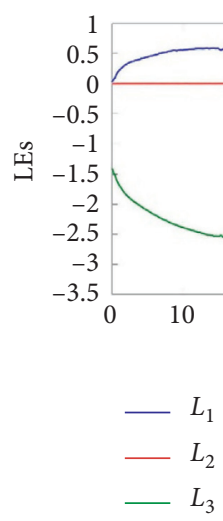

(c)

Figure 5: (a) The bifurcation of the AMWO system with $\alpha$ (red dots showing backward continuation and blue dots showing forward continuation); (b) LEs of forward continuation; (c) LEs of backward continuation.

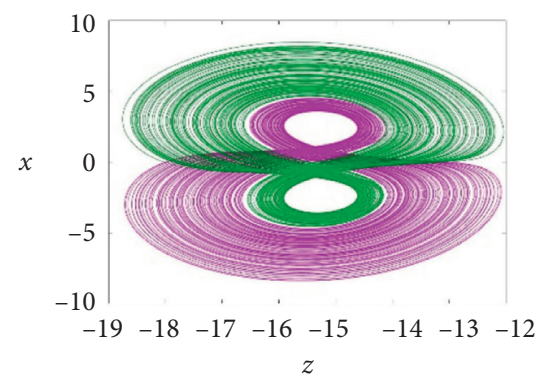

(a)

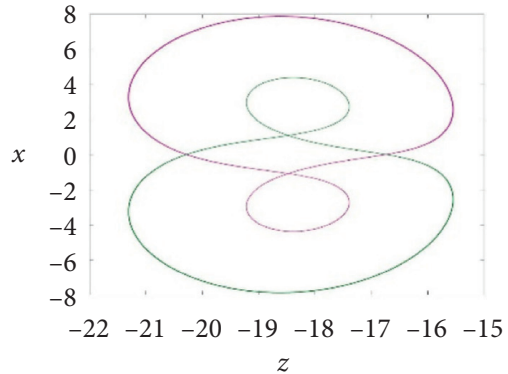

(b)

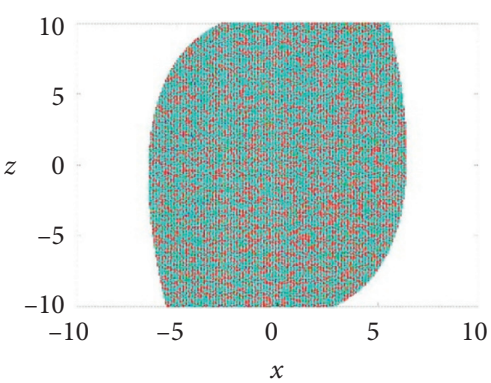

(d)

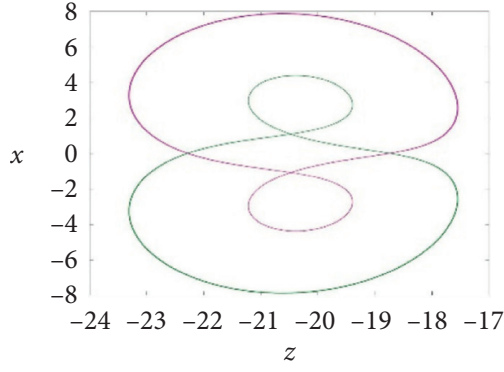

(c)

FIGURE 6: Coexisting attractors of the AMWO for $q=0.98$ and initial conditions $[0.1 ; 0.1 ; 0]$ shown in green colour and $[-0.1 ;-0.1 ; 0]$ shown in purple for (a) $\alpha=60$; (b) $\alpha=72$; (c) $\alpha=80$. (d) The basin of attraction of the AMWO in the x-z plane having the cross-section at $y=0.1$. 


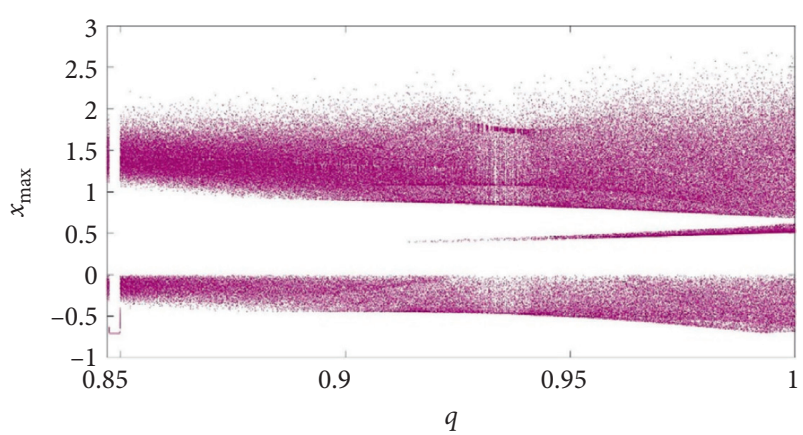

$q$

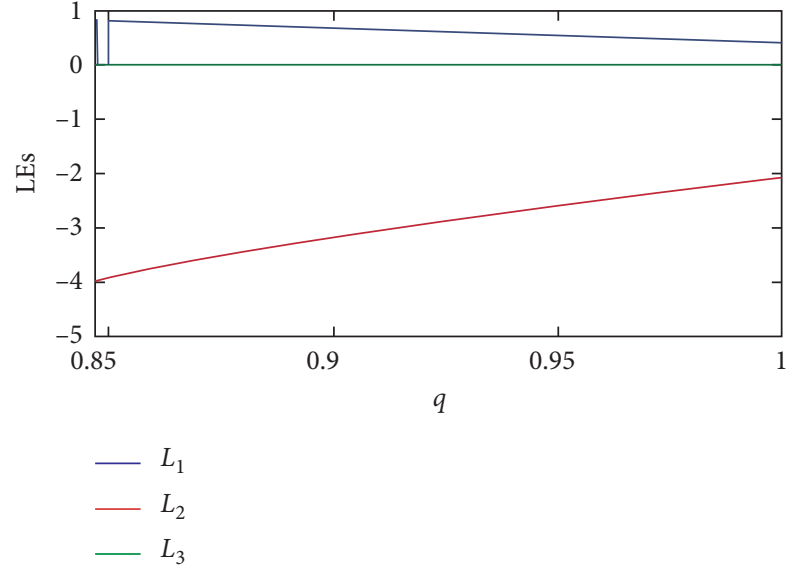

(b)

FIgURE 7: (a) Bifurcation of the AMWO with fractional order q; (b) the corresponding LEs.

consisting of $C_{a}, C_{b}, R_{a}$, and $R_{b}$. For the value of system (5) selected as $a_{1}=4.5, a_{2}=5.5, a_{3}=0.4, a_{4}=1, \alpha=5$, and $\beta=$ 4 and fractional order $q=0.98$, the corresponding values of circuit elements can be calculated as $R_{1}=R_{2}=2.222 \mathrm{k} \Omega$, $R_{3}=2 \mathrm{k} \Omega, \quad R_{4}=2.5 \mathrm{k} \Omega, \quad R_{5}=R_{6}=1.818 \mathrm{k} \Omega, \quad R_{7}=5 \mathrm{k} \Omega$, $R_{8}=25 \mathrm{k} \Omega, \quad R=10 \mathrm{k} \Omega, \quad R_{a}=91.187 \mathrm{M} \Omega, \quad R_{b}=190.933 \Omega$, $C=10 n F, C_{a}=0.9732 \mu \mathrm{F}$, and $C_{b}=3.68059 \mu \mathrm{F}$.

The $2 \mathrm{D}$ phase portraits of the AMWO system implemented in Pspice are shown in Figure 9.

The coexistence of attractors in the AMWO system obtained from the Pspice is presented in Figure 10.

From Figures 9 and 10, we can observe that the Pspicebased circuit simulations' results are consistent with those obtained from numerical simulations (see Figures 3 and 6). This means that the proposed analogue circuit of Figure 8 is able to mimic the dynamics of the AMWO system 5.

\section{Combination Synchronization of the Wien Bridge Oscillator with the Fractional- Order Memristor}

Chaos synchronization plays a very important role in secure communication systems. In recent decades, many secure communication systems based on the synchronization of the one drive system with a response system have been intensively investigated both theoretically and practically using many different methods. However, this type of secure communication systems is relatively vulnerable to the attacks due to the fact that they use only one transmitter. To overcome this problem of security and ensure safer communication, some interesting methods of synchronization have been developed including combination-combination synchronization, multiswitching combination synchronization, combined projective synchronization, equal combination synchronization, and combination synchronization [53-58]. The latter synchronization method consists of using more than one driver systems to synchronize with one response system. This synchronization method has great advantages (e.g., stronger antiattack ability and antitranslated capability) over the traditional drive-response synchronization systems. The combination synchronization method is described as follows: (a) the drive systems are divided into at least two groups, and each group exists as one of the parallel branches in the system combination; (b) the drive systems on the same branch jointly drive the response system; (c) the drive systems on different branches synchronize with the response system separately; (d) the response system takes merely one controller in the combination synchronization.

According to the advantages of such synchronization methods, in this section, we design and perform numerically the synchronization of two drives and one-response absolute memfractor Wien bridge oscillators (AMWO) based on the combination method. The drive-response systems are constructed as

$$
\begin{aligned}
\frac{d x_{m}}{d t} & =a_{1}\left(x_{m}-y_{m}\right)+\left(\alpha_{f}-\beta_{f}\left|z_{m}\right| x_{m}\right), \\
\frac{d y_{m}}{d t} & =a_{2}\left(x_{m}-y_{m}\right), \\
\frac{d^{q} z_{m}}{d t^{q}} & =-a_{3} z_{m}-a_{4} x_{m}^{2},
\end{aligned}
$$

where $m=1$ and 2 and

$$
\begin{aligned}
\frac{d x_{s}}{d t} & =a_{1}\left(x_{s}-y_{s}\right)+\left(\alpha_{f}-\beta_{f}\left|z_{s}\right| x_{s}\right)+u_{1}, \\
\frac{d y_{s}}{d t} & =a_{2}\left(x_{s}-y_{s}\right)+u_{2}, \\
\frac{d^{q} z_{s}}{d t^{q}} & =-a_{3} z_{s}-a_{4} x_{s}^{2}+u_{3},
\end{aligned}
$$

where $u_{i}(i=1,2,3)$ are the control functions to be determined such that the synchronization between the three systems can be realized. For the combination synchronization, the error is defined as

$$
e=A x+B y-C z
$$

where $x=\left(x_{1}, y_{1}, z_{1}\right)^{T}, y=\left(x_{2}, y_{2}, z_{2}\right)^{T}, z=\left(x_{s}, y_{s}, z_{s}\right)^{T}$, and $e=\left(e_{x}, e_{y}, e_{z}\right)^{T}$ and $A, B, C \in \in R^{3 \times 3}$. The main objective is to design the control functions $u_{i}(i=1,2,3)$ for the response 

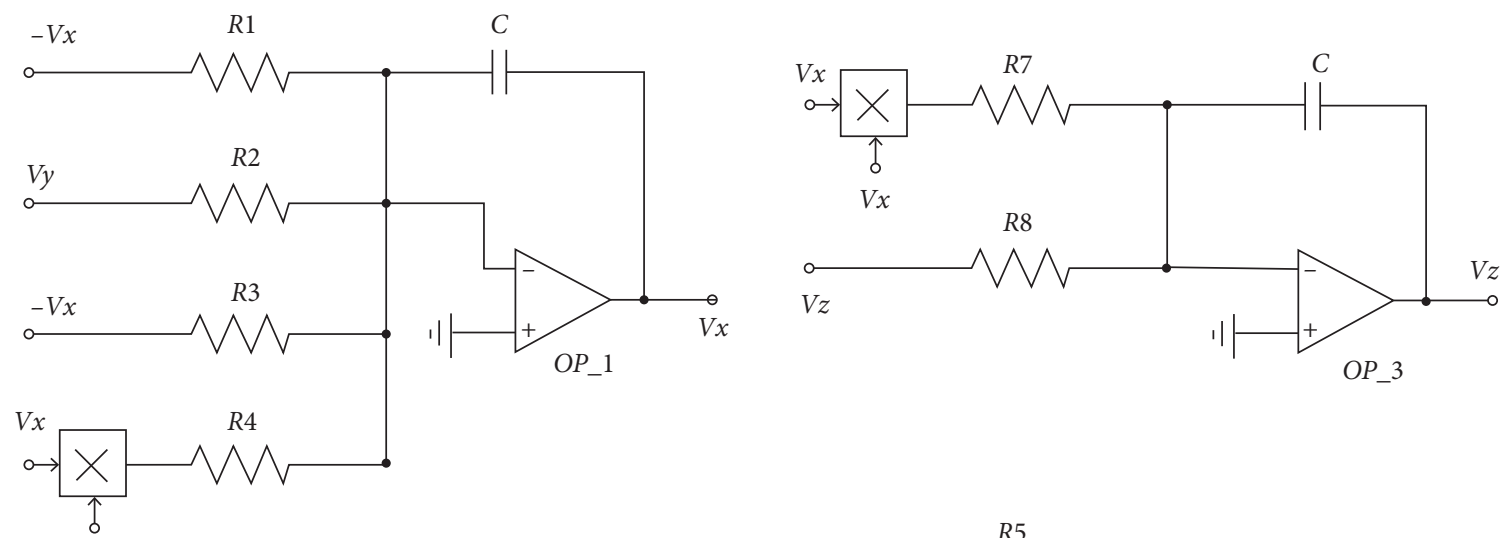

$|V z|$
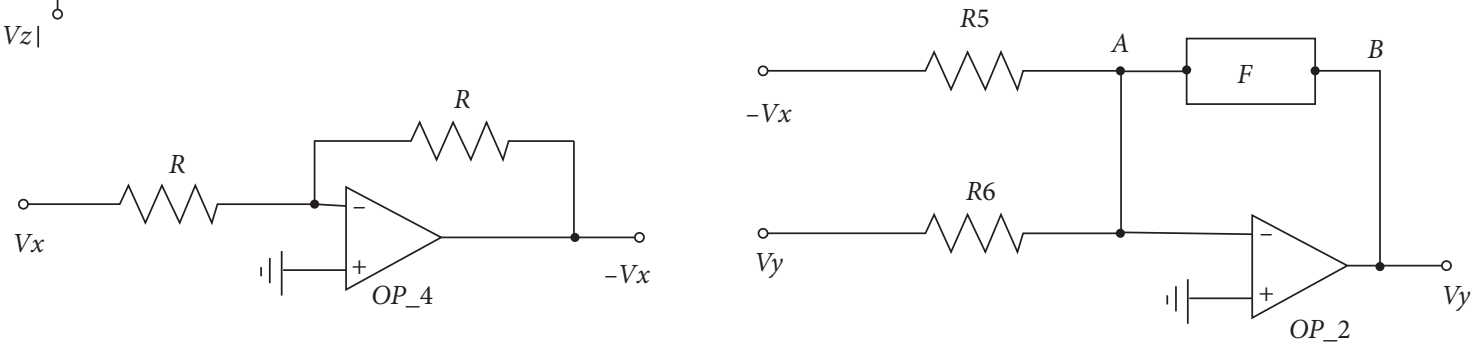

(a)

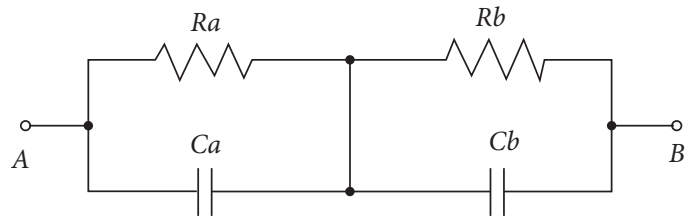

(b)

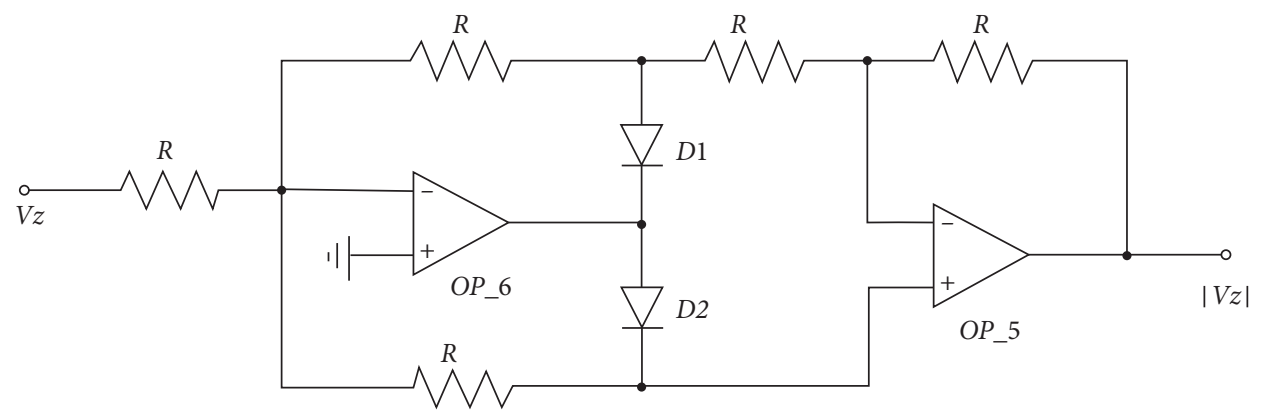

(c)

Figure 8: Analogue circuit diagram modelling of the AMWO system (a). The fractional-order unit circuit (b). Circuit realization of the absolute value function (c).

system [15], such that the error defined by (17) can be asymptotically stable at the zero equilibrium, i.e., $\lim _{t \rightarrow \infty}\|e\|=0$. Thus, the combination synchronization is achieved between the three drive-response systems. To achieve this, we assume that $A=\operatorname{diag}\left(\eta_{1}, \eta_{2}, \eta_{3}\right)$, $B=\operatorname{diag}\left(\gamma_{1}, \gamma_{2}, \gamma_{3}\right)$, and $C=\operatorname{diag}\left(\varepsilon_{1}, \varepsilon_{2}, \varepsilon_{3}\right)$. Considering these assumptions, system (17) is rewritten as

$$
\begin{aligned}
& e_{x}=\eta_{1} x_{1}+\gamma_{1} x_{2}-\varepsilon_{1} x_{s}, \\
& e_{y}=\eta_{2} y_{1}+\gamma_{2} y_{2}-\varepsilon_{2} y_{s}, \\
& e_{z}=\eta_{3} z_{1}+\gamma_{3} z_{2}-\varepsilon_{3} z_{s} .
\end{aligned}
$$

Differentiating (18), we obtain the error dynamical system as 

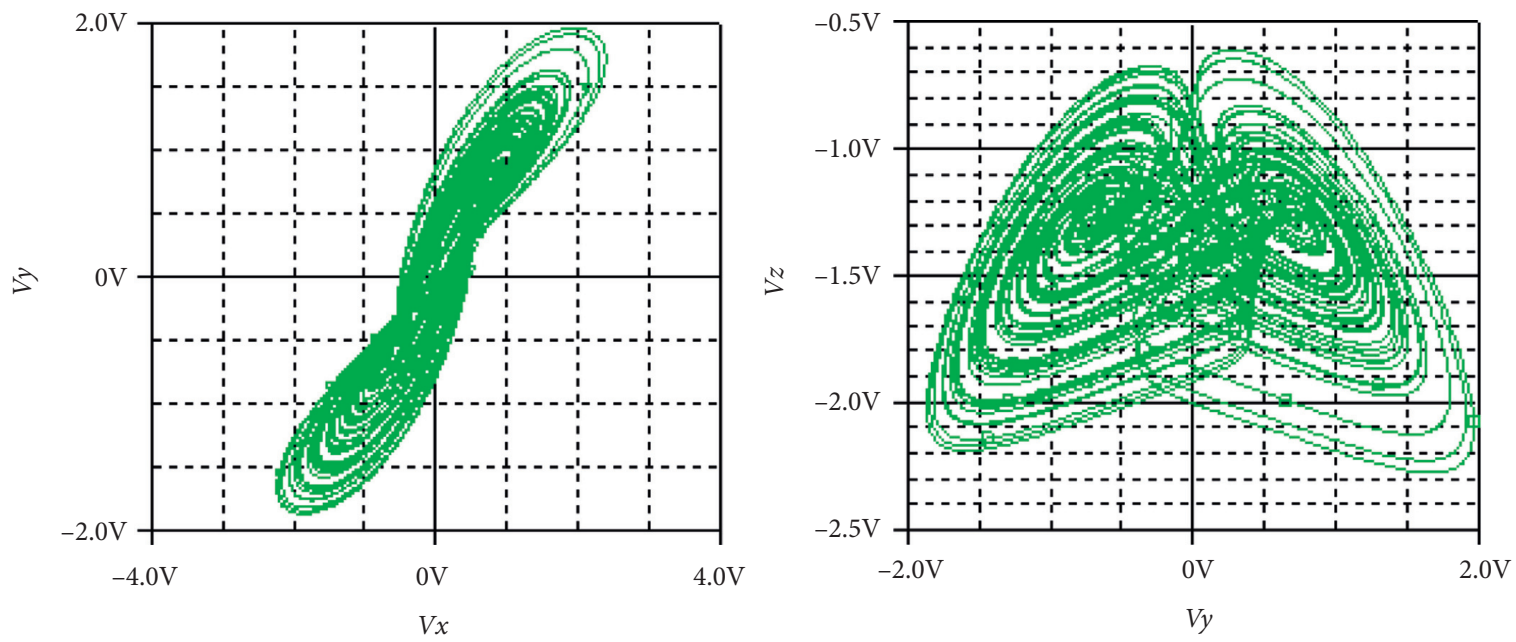

$\square V(Z)$

Figure 9: 2D phase portraits of the absolute memfractor Wien bridge oscillator (AMWO) obtained from Pspice with initial voltages $V_{x}=V_{y}=V_{z}=0.1 V$.

$$
\begin{aligned}
& \frac{d e_{x}}{d t}=\eta_{1} \frac{d x_{1}}{d t}+\gamma_{1} \frac{d x_{2}}{d t}-\varepsilon_{1} \frac{d x_{s}}{d t}, \\
& \frac{d e_{y}}{d t}=\eta_{2} \frac{d y_{1}}{d t}+\gamma_{2} \frac{d y_{2}}{d t}-\varepsilon_{2} \frac{d y_{s}}{d t}, \\
& \frac{d^{q} e_{z}}{d t^{q}}=\eta_{3} \frac{d^{q} z_{1}}{d t^{q}}+\gamma_{3} \frac{d^{q} z_{2}}{d t^{q}}-\varepsilon_{3} \frac{d^{q} z_{s}}{d t^{q}} .
\end{aligned}
$$$$
\text { Replacing systems (15), (16), and (18) into system (19) }
$$
yields

$$
\begin{aligned}
\frac{d e_{x}}{d t} & =\left(a_{1}+\alpha_{f}\right) e_{x}-a_{1}\left(\eta_{1} y_{1}+\gamma_{1} y_{2}-\varepsilon_{1} y_{s}\right)-\beta_{f}\left(\eta_{1}\left|z_{1}\right| x_{1}+\gamma_{1}\left|z_{2}\right| x_{2}-\varepsilon_{1}\left|z_{s}\right| x_{s}\right)-\varepsilon_{1} u_{1}, \\
\frac{d e_{y}}{d t} & =a_{2}\left(\eta_{2} x_{1}+\gamma_{2} x_{2}-\varepsilon_{2} x_{s}\right)-a_{2} e_{y}-\varepsilon_{2} u_{2}, \\
\frac{d^{q} e_{z}}{d t^{q}} & =-a_{3} e_{z}-a_{4}\left(\eta_{3} x_{1}^{2}+\gamma_{3} x_{2}^{2}-\varepsilon_{3} x_{s}^{2}\right)-\varepsilon_{3} u_{3} .
\end{aligned}
$$

The control functions are derived from system (20) as follows:

$$
\begin{aligned}
& u_{1}=\frac{\left(-a_{1}\left(\eta_{1} y_{1}+\gamma_{1} y_{2}-\varepsilon_{1} y_{s}\right)-\beta_{f}\left(\eta_{1}\left|z_{1}\right| x_{1}+\gamma_{1}\left|z_{2}\right| x_{2}-\varepsilon_{1}\left|z_{s}\right| x_{s}\right)-v_{x}\right)}{\varepsilon_{1}} \\
& u_{2}=\frac{\left(a_{2}\left(\eta_{2} x_{1}+\gamma_{2} x_{2}-\varepsilon_{2} x_{s}\right)-v_{y}\right)}{\varepsilon_{2}} \\
& u_{3}=\frac{\left(-a_{4}\left(\eta_{3} x_{1}^{2}+\gamma_{3} x_{2}^{2}-\varepsilon_{3} x_{s}^{2}\right)-v_{z}\right)}{\varepsilon_{3}}
\end{aligned}
$$



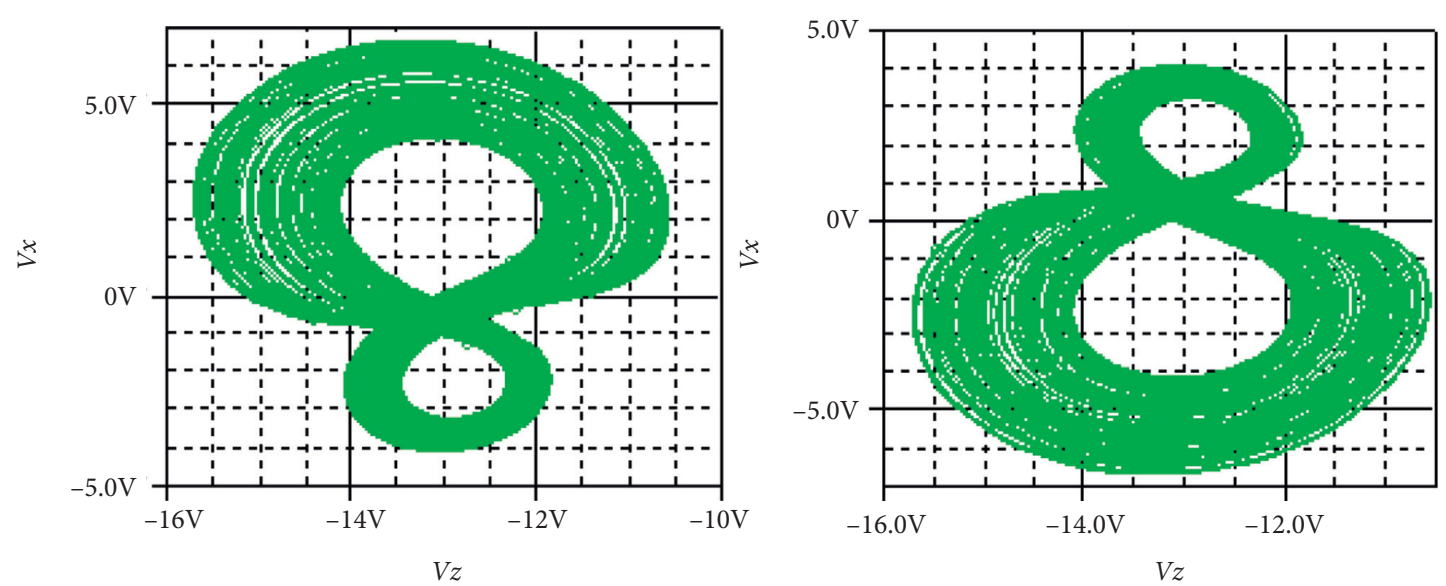

(a)
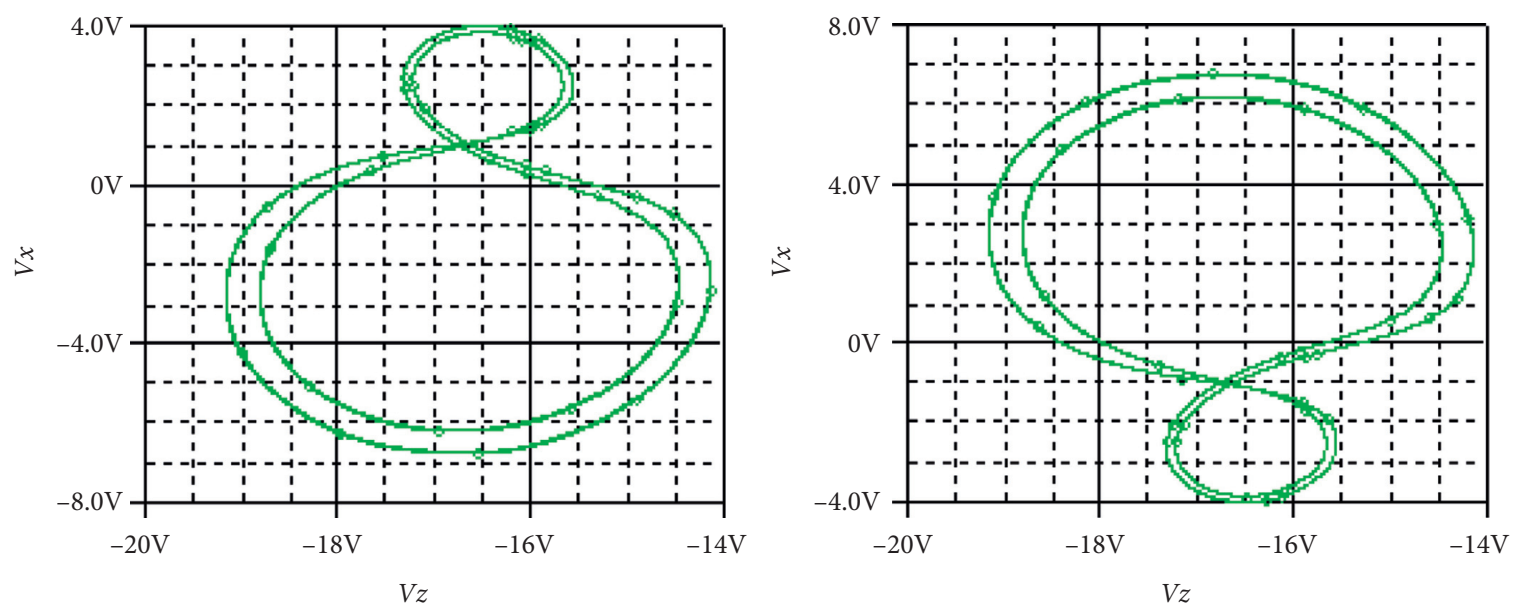

(b)
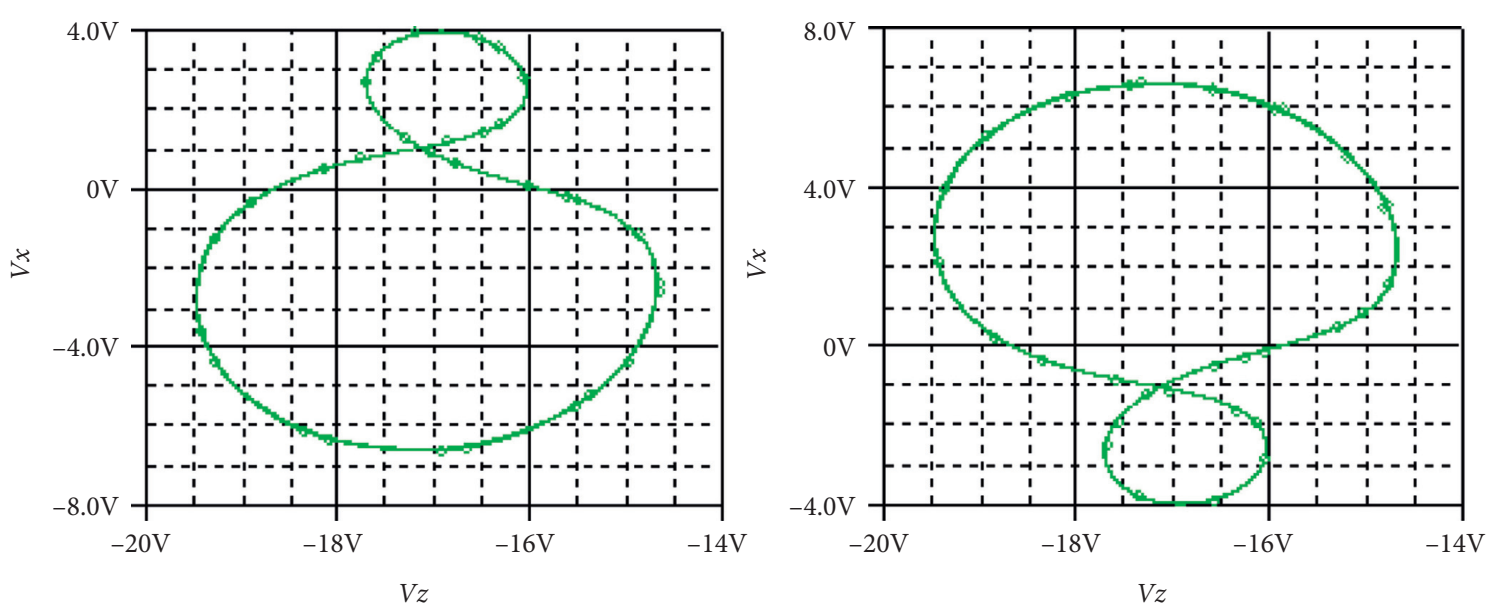

(c)

FIgURe 10: Coexisting attractors of the AMWO obtained from Pspice with the initial voltages $V_{x}=V_{y}= \pm 0.1 \mathrm{~V}$ and $V_{z}=0.0 \mathrm{~V}$ for (a) $R_{3}=166.666 \Omega$, (b) $R_{3}=138.888 \Omega$, and (c) $R_{3}=125 \Omega$. The other values of circuit elements are defined as in the text. 


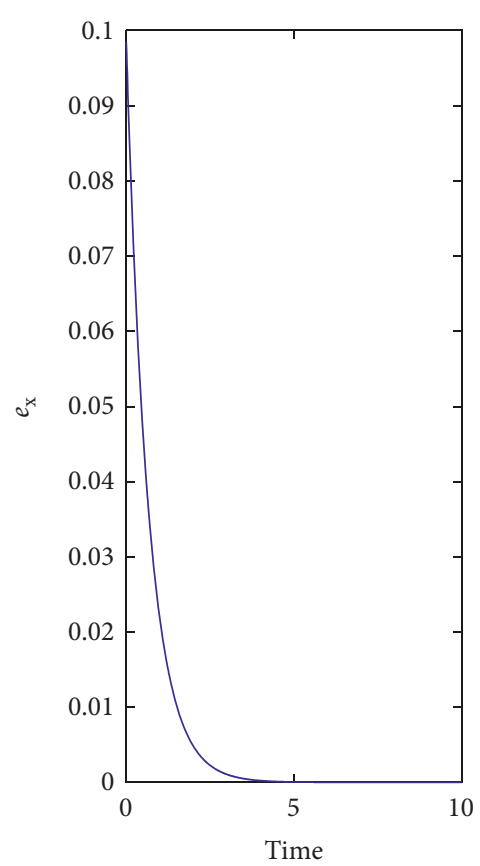

(a)

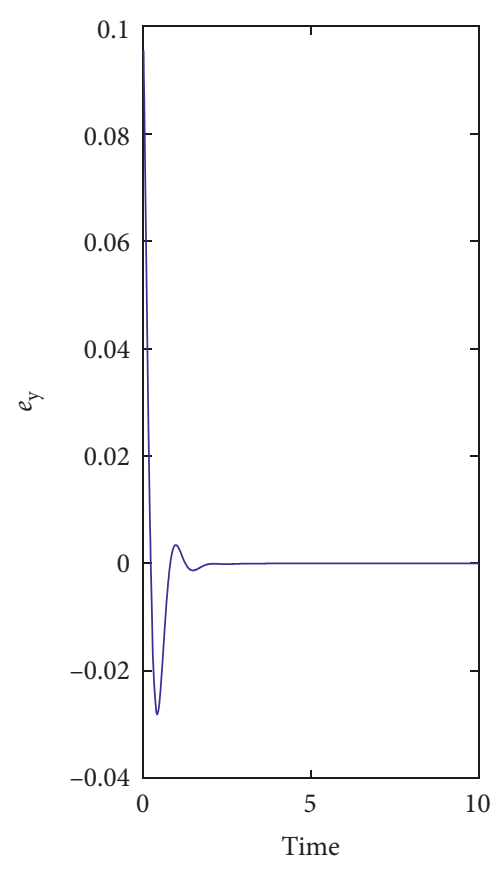

(b)

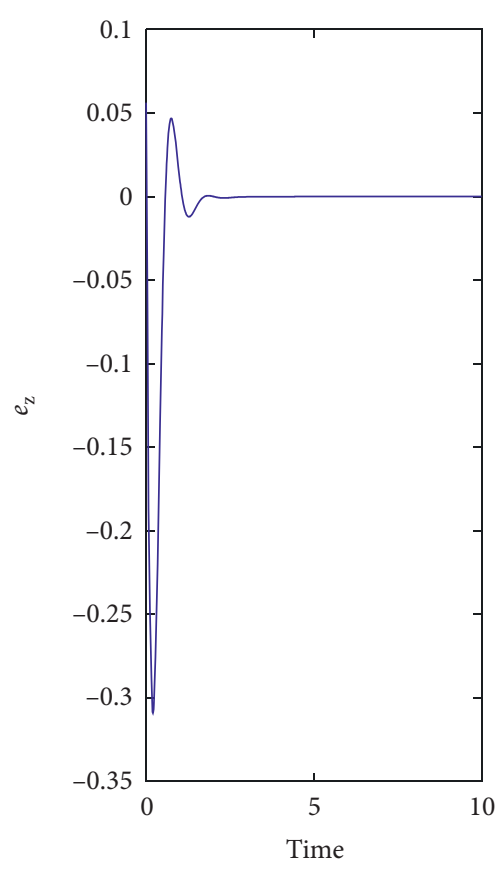

(c)

FIGURE 11: Combination synchronization errors between drive (1) and response (2) absolute memfactor Wien bridge oscillators for $a_{1}=4.5$, $a_{2}=5.5, a_{3}=0.4, \alpha_{f}=5, \beta_{f}=4$, and $q=0.98$, and the control parameters are selected as $\eta_{i}=\gamma_{i}=\varepsilon_{i}=1(i=1,2,3)$.

in which $v_{i}(i=x, y, w)$ are linear functions selected such that the error dynamical system becomes stable. Lets choose these linear functions in the following form:

$$
\left(\begin{array}{c}
v_{x} \\
v_{y} \\
v_{z}
\end{array}\right)=\bar{A}\left(\begin{array}{c}
e_{x} \\
e_{y} \\
e_{z}
\end{array}\right),
$$

where

$$
\bar{A}=\left(\begin{array}{lll}
a_{11} & a_{12} & a_{13} \\
a_{21} & a_{22} & a_{23} \\
a_{31} & a_{32} & a_{33}
\end{array}\right)
$$

is a $3 \times 3$ real matrix.

For $a_{11}=-11, a_{12}=0, a_{13}=0, a_{21}=0, a_{22}=0, a_{23}=1$, $a_{31}=-1.5, a_{33}=0, a_{31}=-1.5, a_{1}=4.5, a_{2}=5.5, a_{3}=0.4$, and $\alpha_{f}=5$, the error dynamical system becomes

$$
\begin{aligned}
& \frac{d e_{x}}{d t}=\left(a_{1}+\alpha_{f}-11\right) e_{x}, \\
& \frac{d e_{y}}{d t}=-a_{2} e_{y}+e_{z}, \\
& \frac{d^{q} e_{z}}{d t^{q}}=-1.5 e_{x}-38 e_{y}-a_{3} e_{z} .
\end{aligned}
$$

Note that these values of parameters are chosen such that the error dynamical system becomes stable.

For $a_{4}=1, \beta_{f}=4$, and $q=0.98$, the eigenvalues of error dynamical system (24) are $\lambda_{1}=-1.5, \lambda_{2}=-2.95+5.6123 j$, and $\lambda_{3}=-2.95-5.6123 j$. The condition for the stability of the error dynamical system (24) is $\left|\arg \left(\lambda_{i}\right)\right|>q \pi / 2$. Using the above eigenvalues, we obtain $\left|\arg \left(\lambda_{i}\right)\right|=2.0547$ which satisfy the above condition for stability. This means that the error states converge asymptotically to zero as $t \longrightarrow \infty$, and thus, the combination synchronization between the three driveresponse systems is achieved.

For numerical verifications, we fix $a_{1}=4.5, a_{2}=5.5$, $a_{3}=0.4, a_{4}=1, \alpha_{f}=5, \beta_{f}=4$, and $q=0.98$ such that the AMWO system is still chaotic. The initial states of drive and response systems (15) and (16) are chosen, respectively, as $x_{1}(0)=y_{1}(0)=z_{1}(0)=0.1, \quad x_{2}(0)=2, \quad y_{2}(0)=-1$, $z_{2}(0)=0.1, \quad x_{s}(0)=1, \quad y_{s}(0)=0.3$, and $z_{s}(0)=2$. The combination synchronization results depicting the errors between the drive and response systems (15) and (16) are presented in Figure 8. From Figure 11, one can observe that the error states converge to zero which implies that the combination synchronization is realized between the three drive-response systems (15) and (16).

\section{Conclusion}

In this paper, we proposed a Wien bridge oscillator with the fractional-order memristor. Most of the fractional-order Wien bridge oscillators consider all the states as noninteger orders, but in this paper, we considered only the internal state of the memristor as the fractional order which enables us to explore a wide variety of unexplored properties like the multistability and coexisting attractors. Pspice-based circuit simulations are shown to prove the realizability of the proposed oscillator. The results of the Pspice simulations are compared with theoretical results. A combination 
synchronization scheme is proposed in which we have used two slave systems to synchronize with the master system. Such techniques enable us to implement much complex antiattack features by using two different methods. The first one consists of splitting the transmitted signals into two parts where each part is loaded in different drive systems, while the second one consists of dividing time into different intervals and loading the signals in different drive systems. The synchronization errors are numerically calculated and are shown to prove the effectiveness of the proposed technique.

\section{Data Availability}

All the numerical simulation parameters are included within the article, and there were no additional data requirements for the simulation results.

\section{Conflicts of Interest}

The authors declare that they have no conflicts of interest.

\section{References}

[1] Q. Lai, A. Akgul, C. Li, G. Xu, and U. Cavusoglu, "A new chaotic system with multiple attractors: dynamic analysis, circuit realization and S-Box design," Entropy, vol. 20, no. 1, p. 12, 2018.

[2] C. Li, "Homogenous multistability in memristive system, new trends in nonlinear dynamics," in Proceedings of the First International Nonlinear Dynamics Conference (NODYCON 2019, vol. III, Rome, Italy, February 2019.

[3] Q. Lai, Z. Wan, P. D. Kamdem Kuate, and H. Fotsin, "Coexisting attractors, circuit implementation and synchronization control of a new chaotic system evolved from the simplest memristor chaotic circuit," Communications in Nonlinear Science and Numerical Simulation, vol. 89, p. 105341, 2020.

[4] T. Lu, C. Li, X. Wang, C. Tao, and Z. Liu, "A memristive chaotic system with offset-boostable conditional symmetry," The European Physical Journal Special Topics, vol. 229, pp. 1059-1069, 2020.

[5] Z. Wei, V. T. Pham, A. J. M. Khalaf, J. Kengne, and S. Jafari, “A modified multistable chaotic oscillator," International Journal of Bifurcation and Chaos, vol. 28, no. 7, 2018.

[6] Q. Lai, P. D. Kamdem Kuate, F. Liu, and H. H.-C. Iu, "An extremely simple chaotic system with infinitely many coexisting attractors," IEEE Transactions on Circuits and Systems II: Express Briefs, vol. 67, no. 6, pp. 1129-1133, 2020.

[7] Z. Wei, P. Yu, W. Zhang, and M. Yao, "Study of hidden attractors, multiple limit cycles from Hopf bifurcation and boundedness of motion in the generalized hyperchaotic Rabinovich system," Nonlinear Dynamics, vol. 82, pp. 131$141,2015$.

[8] Q. Lai, Z. Wan, and P. D. Kamdem Kuate, "Modelling and circuit realisation of a new no-equilibrium chaotic system with hidden attractor and coexisting attractors," Electronics Letters, vol. 56, no. 20, p. 1044, 2020.

[9] Z. Wei, I. Moroz, J. C. Sprott, Z. Wang, and W. Zhang, "Detecting hidden chaotic regions and complex dynamics in the self-exciting homopolar disc dynamo," International Journal of Bifurcationand Chaos in Applied Sciences and Engineering, vol. 27, no. 2, 2017.
[10] Z. Wei, I. Moroz, J. C. Sprott, A. Akgul, and W. Zhang, "Hidden hyperchaos and electronic cirapplication in a 5D self-exciting homopolar disc dynamo," Chaos, vol. 27, no. 2, 2017.

[11] Z. Wei, Viet-Thanh Pham, T. Kapitaniak, and Z. Wang, "Bifurcation analysis and circuit realization for multipledelayed Wang-Chen system with hidden chaotic attractors," Nonlinear Dynamics, vol. 85, pp. 1635-1650, 2016.

[12] Z. Wei, B. Zhu, J. Yang, M. Perc, and M. Slavinec, "Bifurcation analysis of two disc dynamos with viscous friction and multiple time delays," Applied Mathematics and Computation, vol. 347, pp. 265-281, 2019.

[13] Y. Li, Z. Wei, W. Zhang, M. Perc, and R. Repnik, "BogdanovTakens singularity in the Hindmarsh-Rose neuron with time delay," Applied Mathematics and Computation, vol. 354, pp. 180-188, 2019.

[14] Q. Lai, B. Norouzi, and F. Liu, "Dynamic analysis, circuit realization, control design and image encryption application of an extended Lü system with coexisting attractors," Chaos, Solitons \& Fractals, vol. 114, pp. 230-245, 2018.

[15] S. He, K. Sun, Y. Peng, and L. Wang, "Modeling of discrete fracmemristor and its application," AIP Advances, vol. 10, no. 1, p. 015332, 2020.

[16] S. He, K. Sun, and Y. Peng, "Detecting chaos in fractionalorder nonlinear systems using the smaller alignment index," Physics Letters A, vol. 383, no. 19, p. 2267, 2019.

[17] S. He, K. Sun, H. Wang, X. Mei, and Y. Sun, "Generalized synchronization of fractional-order hyperchaotic systems and its DSP implementation," Nonlinear Dynamics, vol. 92, no. 1, pp. 85-96, 2018.

[18] O. P. Agrawal, J. A. Tenreiro-Machado, and I. Sabatier, Fractional Derivatives and Their Applications" Nonlinear Dynamics, Vol. 38, Springer-Verlag, Berlin, Germany, 2004.

[19] R. Hilfer, Applications of Fractional Calculus in Physics, World Scientific, Singapore, 2000.

[20] B. J. West, M. Bologna, and P. Grigolini, Physics of Fractional Operators, Springer-Verlag, Berlin, Germany, 2003.

[21] R. L. Magin, Fractional Calculus in Bioengineering, Begell House Publisher, Rodding, Denmark, 2006.

[22] E. Ryabov Ya and A. Puzenko, "Damped oscillations in view of the fractional oscillator equation," Physical Review B, vol. 66, no. 18, 2002.

[23] M. Naber, "Linear fractionally damped oscillator," International Journal of Differential Equations, vol. 2010, Article ID 197020, 12 pages, 2010.

[24] I. Petras, Y. Q. Chen, and C. Coopmans, "Fractional-order memristive systems,", in Proceedings of the 2009 IEEE Conference on Emerging Technologies \& Factory Automation, pp. 1-8, Mallorca, Spain, September 2009.

[25] I. Petras and Y. Q. Chen, "Fractional-order circuit elements with memory," in Proceedings of the 13th International Carpathian Control Conference (ICCC), pp. 552-558, HighTatras, Slovakia, May 2012.

[26] N. Yang, C. Xu, C. Wu, R. Jia, and C. Liu, "Modeling and analysis of a fractional-order generalized memristor-based chaotic system and circuit implementation," International Journal of Bifurcation and Chaos, vol. 27, no. 13, 2017.

[27] F. Z. Wang, L. Shi, H. Wu, N. Helian, and L. O. Chua, "Fractional memristor," Applied Physics Letters, vol. 111, no. 24, 2017.

[28] I. Petras, "Fractional-order memristor-based Chua's circuit," IEEE Transactions on Circuits and Systems II: Express Briefs, vol. 57, no. 12, pp. 975-979, 2010. 
[29] H. H. C. Iu, D. S. Yu, A. L. Fitch, V. Sreeram, and H. Chen, "Controlling chaos in a memristor based circuit using a twinT notch filter," IEEE Transactions on Circuits and Systems I: Regular Papers, vol. 58, no. 6, pp. 1337-1344, 2011.

[30] B. Muthuswamy, "Implementing memristor based chaotic circuits," International Journal of Bifurcation and Chaos, vol. 20, no. 5, pp. 1335-1350, 2010.

[31] A. Buscarino, L. Fortuna, M. Frasca, and L. V. Gambuzza, "A chaotic circuit based on Hewlett-Packard memristor," Chaos, vol. 22, no. 2, 2012.

[32] A. L. Fitch, D. Yu, H. H. C. Iu, and V. Sreeram, "Hyperchaos in a memristor-based modified canonical chua's circuit," International Journal of Bifurcation and Chaos, vol. 22, no. 6, p. 1250133, 2012.

[33] R. Rizwana and I. Raja Mohamed, "Investigation of chaotic and strange nonchaotic phenomena in nonautonomous wienbridge oscillator with diode nonlinearity," Journal of Nonlinear Dynamics, vol. 2015, Article ID 612516, 7 pages, 2015.

[34] H. Wu, B. Bao, Z. Liu, Q. Xu, and P. Jiang, "Chaotic and periodic bursting phenomena in a memristive Wien-bridge oscillator," Nonlinear Dynamics, vol. 83, no. 1-2, pp. 893-903, 2015.

[35] X. Ye, X. Wang, J. Mou, X. Yan, and Y. Xian, "Characteristic analysis of the fractional-order hyperchaotic memristive circuit based on the Wien bridge oscillator," The European Physical Journal Plus, vol. 133, p. 516, 2018.

[36] Q. Xu, Q. Zhang, T. Jiang, B. Bao, and M. Chen, "Chaos in a second-order non-autonomous Wien-bridge oscillator without extra nonlinearity," Circuit World, vol. 44, no. 3, 2018.

[37] N. Yang, C. Xu, C. Wu, R. Jia, and C. Liu, "Dynamic behaviors and the equivalent realization of a novel fractional-order memristor-based chaotic circuit"' Complexity, vol. 2018, p. 13, Article ID 9467435, 2018.

[38] Z. Wang, M. Yin, T. Zhang et al., "Engineering incremental resistive switching in TaOxbased memristors for brain-inspired computing," Nanoscale, vol. 8, no. 29, p. 14015, 2016.

[39] J. Chen, Z. Zeng, and P. Jiang, "Global Mittag-Leffler stability and synchronization of memristor-based fractional-order neural networks," Neural Networks, vol. 51, no. -, pp. 1-8, 2014.

[40] L. Chen, Y. Chai, R. Wu, T. Ma, and H. Zhai, "Dynamic analysis of a class of fractional-order neural networks with delay," Neurocomputing, vol. 111, pp. 190-194, 2013.

[41] J. Luo, X. Xu, Y. Ding et al., "Application of a memristorbased oscillator to weak signal detection," The European Physical Journal Plus, vol. 133, p. 239, 2018.

[42] H.-H. Peng, X.-M. Xu, B.-C. Yang, and L.-Z. Yin, "Implication of two-coupled differential van der Pol duffing oscillator in weak signal detection," Journal of the Physical Society of Japan, vol. 85 , no. $4,2016$.

[43] M.-S. Abdelouahab, R. Lozi, and L. Chua, "Memfractance: A mathematical paradigm for circuit elements with memory," International Journal of Bifurcation and Chaos, vol. 24, no. 9, p. 1430023, 2014.

[44] K. Rajagopal, C. Li, F. Nazarimehr, A. Karthikeyan, P. Duraisamy, and S. Jafari, "Chaotic Dynamics of a Simple Wein Bridge Oscillator with Fractional Order Memristor," Radioengineering, vol. 27, no. 1, pp. 165-174, 2019.

[45] C. Muñiz-Montero, L. V. García-Jiménez, L. A. SánchezGaspariano, C. Sánchez-López, V. R. González-Díaz, and E. Tlelo-Cuautle, "New alternatives for analog implementation of fractional-order integrators, differentiators and PID controllers based on integer-order integrators," Nonlinear Dynamics, vol. 90, pp. 241-256, 2017.
[46] K. Diethelm, "An algorithm for the numerical solution of differential equations of fractional order," Electronic Transactions on Numerical Analysis ETNA, vol. 5, pp. 1-6, 1998.

[47] K. Diethelm and N. J. Ford, "Analysis of fractional differential equations," Journal of Mathematical Analysis and Applications, vol. 265, no. 2, pp. 229-248, 2002.

[48] H. Sun, A. Abdelwahab, and B. Onaral, "Linear approximation of transfer function with a pole of fractional power," IEEE Transactions on Automatic Control, vol. 29, no. 5, pp. 441-444, 1984.

[49] K. Diethelm and A. D. Freed, "The FracPECE subroutine for the numerical solution of differential equations of fractional order," Forschung und Wissenschaftliches Rechnen, vol. 1999, pp. 57-71, 1998.

[50] A. Wolf, J. B. Swift, H. L. Swinney, and J. A. Vastano, "Determining Lyapunov exponents from a time series," Physica D: Nonlinear Phenomena, vol. 16, no. 3, pp. 285-317, 1985.

[51] R. Garrappa, "Predictor-corrector PECE method for fractional differential equations," MATLAB Central File Exchange, Natick, MA, USA, 2011, https://www.mathworks. com/matlabcentral/fileexchange/32918-predictor-correctorpece-method-for-fractional-differential-equations.

[52] M. F. Danca, "Lyapunov exponents of a class of piecewise continuous systems of fractional order," Nonlinear Dynamics, vol. 81, pp. 227-237, 2015.

[53] X. Zhou, L. Xiong, and X. Cai, "Combination-combination Synchronization of four nonlinear complex chaotic systems," Abstract and Applied Analysis, vol. 2014, no. 5, 14 pages, Article ID 953265, 2014.

[54] U. E. Vincent, A. O. Saseyi, and P. V. E. McClintock, "Multiswitching combination synchronization of chaotic systems," Nonlinear Dynamics, vol. 80, pp. 845-854, 2015.

[55] R. Luo and Y. Zeng, "The equal combination synchronization of a class of chaotic systems with discontinuous output," CHAOS, vol. 25, no. 11, p. 113102, 2015.

[56] C.-F. Feng, Y.-R. Tan, Y.-H. Wang, and H.-J. Yang, “Active backstepping control of combined projective synchronization among different nonlinear systems," Automatika, vol. 58, no. 3, pp. 295-301, 2017.

[57] R. Luo, Y. Wang, and S. Deng, "Combination synchronization of three classic chaotic systems using active backstepping design," Chaos, vol. 21, no. 4, 2011.

[58] J. R. M. Pone, S. T. Kingni, G. R. Kol, and V. T. Pham, "Hopf bifurcation, antimonotonicity and amplitude controls in the chaotic Toda jerk oscillator: analysis, circuit realization and combination synchronization in its fractional-order form," Automatika, vol. 60, no. 2, pp. 149-161, 2019. 